\title{
Isotope-dilution LC-MS/MS Analysis of the Elastin Crosslinkers Desmosine and Isodesmosine in Acute Cerebral Stroke Patients
}

Ayame Mikagi

Sophia University

Ryosuke Tashiro

Tohoku University Graduate School of Medicine

Tomoo Inoue

Sophia University

Riki Anzawa

Sophia University

Akiho Imura

Sophia University

Takahiro Tanigawa

Sophia University

Tomohisa Ishida

Sendai Medical Center

Takashi Inoue

Sendai Medical Center

Kuniyasu Niizuma

Tohoku University

Teiji Tominaga

Tohoku University Graduate School of Medicine

Toyonobu Usuki ( $\nabla$ t-usuki@sophia.ac.jp )

Sophia University

\section{Research Article}

Keywords: Elastin, Desmosine, Isotope, LC-MS/MS, Stroke, Vascular injury

Posted Date: October 1st, 2021

DOI: https://doi.org/10.21203/rs.3.rs-943517/v1 
License: (c) (i) This work is licensed under a Creative Commons Attribution 4.0 International License. Read Full License 


\section{Abstract}

We chemically synthesized an isotopically labeled internal standard, isodesmosine- ${ }^{13} \mathrm{C}_{3},{ }^{15} \mathrm{~N}_{1}$, and established an isotope-dilution LC-MS/MS method. Plasma concentrations of desmosine and isodesmosine in acute cerebral stroke patients and healthy controls were determined. The desmosine concentration was markedly higher in plasma from acute stroke patients compared with healthy controls. Desmosines are novel biomarkers for evaluating the extent of vascular injury after acute cerebral stroke.

\section{Introduction}

Homeostasis of the extracellular matrix is critical for maintaining vascular integrity. The extracellular matrix proteins elastin and collagen are particularly important for the maintenance of vascular walls. Elastin is a major component of the fibers that provide elasticity to the alveoli, skin, ligaments, blood vessels, and other components of the body $[1,2]$. Elastin fibers contribute to the elastic characteristic of the arterial wall, whereas collagen is a principal load-bearing component of vascular walls. Along with collagen accumulation, the elastic fibers augment stiffness and resist degradation and fragmentation induced by various stimuli. However, disruption of the elaborate mechanism regulating elastin homeostasis due to pathological conditions such as chronic obstructive pulmonary disease (COPD) or vascular injury results in irreversible degradation of elastin-containing tissues. These observations suggest elastin has potential value as a biomarker of tissue and vascular damage.

Quantitative measurement of the products of extracellular matrix degradation could enhance our understanding of tissue and vascular injuries. Desmosine and isodesmosine (Fig. 1) are crosslinked pyridinium-based amino acids that exist only in the extracellular matrix protein elastin $[3,4]$. However, the precise measurement of desmosine and isodesmosine is technically challenging. Although deuterated desmosine internal standards derived from natural products are available, these standard compounds are not stable during the acid hydrolysis process required to dissociate desmosine and isodesmosine in tissues. Recent analyses of desmosine and isodesmosine have been developed using liquid chromatography-mass spectrometry (LC-MS) or liquid chromatography-tandem mass spectrometry (LCMS/MS: see Figure S0) [5-13]. Notably, patients with exacerbated COPD exhibit higher urinary and blood desmosine levels than healthy controls [14]. Desmosine and isodesmosine can thus be used as biomarkers to gauge the severity of COPD.

As an isotopically labeled internal standard for LC-MS/MS analyses, a previous study utilized synthetic desmosine- $d_{4}$, in which four deuterium atoms were incorporated into desmosine. Even though isotopically labeled internal standards are among the most favorable compounds for sensitive and accurate quantitative measurements [15], the synthetic desmosine- $\mathrm{d}_{4}$ isotopic standard contained impurities such as desmosine- $d_{2}$ and desmosine- $d_{3}[16,17]$, which led to inaccurate quantitation. Furthermore, heptafluorobutyric acid (HFBA), which is commonly used as an ion pair reagent in separations of compounds with similar polarity, inhibits MS ionization and also carries a risk of 
contamination [18]. Therefore, the development of highly pure isotopically labeled internal standards would enable more-precise quantitation of desmosine and isodesmosine.

In the present study, we chemically synthesized isotopically labeled isodesmosine $-{ }^{13} \mathrm{C}_{3},{ }^{15} \mathrm{~N}_{1}$ (Fig. 1) [19] via praseodymium-promoted Chichibabin pyridinium synthesis, as we reported the total synthesis of desmosine and isodesmosine $[20,21]$. Using isotopically labeled isodesmosine $-{ }^{13} \mathrm{C}_{3},{ }^{15} \mathrm{~N}_{1}$ as an internal control, we established an isotope-dilution LC-MS/MS method. Additionally, the method developed in this work should be free from any ion-pair reagents for routine analysis of desmosines.

Cerebral stroke is a devastating cerebrovascular disorder composed of ischemic stroke (cerebral infarction) and hemorrhagic stroke (intracerebral hemorrhage and subarachnoid hemorrhage) [22-24]. Regardless of stroke subtype, cerebral stroke can result in death or disability, resulting in significant socioeconomic burden worldwide [25]. Despite the remarkable advances in treatment modalities for acute ischemic stroke, such as intravenous thrombolysis and endovascular thrombectomy, the indications of these treatments are limited to acute ischemic stroke due to their relatively small therapeutic windows and the specialized expertise required for endovascular thrombectomy $[22,26]$. With regard to hemorrhagic stroke, surgical interventions are currently performed to reduce mass effects of hemorrhaging and prevent re-bleeding $[27,28]$. However, no effective treatment is available to reduce secondary brain injuries resulting from cell death, inflammation, oxidative stress, vascular injuries, and brain edema $[22,29,30]$. In particular, vascular injury caused by inflammation and oxidative stress is a primary mechanism of brain injury, as vascular injuries worsen brain edema and enhance inflammation $[22,29,30]$. Although biomarkers of oxidative stress and inflammation have been developed, there are no useful biomarkers available to detect vascular injuries after acute cerebral stroke [31,32].

In the present study, we hypothesized that products of extracellular matrix degradation would be useful biomarkers of vascular injury after acute cerebral stroke. Specifically, we hypothesized that quantification of desmosine and isodesmosine levels could be used to detect vascular injuries after acute cerebral stroke. The concentrations of desmosine and isodesmosine were measured in acute cerebral stroke patients and healthy controls using an isotope-dilution LC-MS/MS method.

\section{Results And Discussion}

\section{Establishment of the isotopically labeled LC-MS/MS method}

A Shimadzu LCMS-8030 plus system, which exhibits high sensitivity and good reproducibility, was used for LC-MS/MS analyses. The initial attempt to select an appropriate high-performance liquid chromatography (HPLC) column focused on Waters Symmetry C18 and YMC-Pack ODS-AM, which are typical octadecylsilane (ODS) columns. A gradient system of water-acetonitrile (MeCN) was utilized for the elution solvent. As trifluoroacetic acid is less and might have some effects on the MS instruments, $0.1 \%(\mathrm{v} / \mathrm{v})$ formic acid (FA) was used to enhance both column retention and ionization during MS. 
However, the reproducibility of the desmosine and isodesmosine peaks was not acceptable. Alternatively, we used a Supelco Discovery HS F5-3 pentafluorophenyl (F5)-type column, which exhibits unique selectivity characteristics due to p-p stacking interactions with aromatic compounds, delocalized electron density induced by fluorine, and weaker hydrogen bonds [33]. Using this column, the reproducibility of desmosine and isodesmosine peaks was improved using a water-acetonitrile gradient system with the addition of $0.1 \%(\mathrm{v} / \mathrm{v})$ FA without an ion pairing reagent such as HFBA (Table S1). However, chromatographic separation of desmosine and isodesmosine was not accomplished under any LC conditions.

We then revised our strategy to optimize the tandem MS/MS method and establish appropriate equations for calculations. Major factors that can affect analytical sensitivity include selection of ions and the voltage of each MS compartment [34]. Optimizations were performed for general parameters, including the voltage of MS compartments with flow injection without a column. The program began with determination of the precursor ion followed by product ion search, which enumerates desirable product ions. The precursor ion was set according to the molecular weight of desmosine. A protonated doublecharged ion $(\mathrm{m} / \mathrm{z} 263.25)$ was selected as the precursor ion, and product ions exhibiting high intensity were selected in the second step. Voltage optimization was performed for these precursor and product ions automatically [35].

Based on observed $\mathrm{m} / \mathrm{z}$ values and the structures of desmosine and isodesmosine, the structures of some fragment ions were estimated (Figure S1) using multi-reaction monitoring (MRM) mode. It should be noted that isodesmosine formed the same fragments, but the relative intensity of the ions enabled them to be distinguished from desmosine [36]. The $m / z 232.10$ and 397.25 ions were clearly different; the $\mathrm{m} / z 232.10$ ion was easier to detect than the $m / z 397.25$ ion from desmosine. However, in the case of isodesmosine, the area of the $m / z 397.25$ ion peak was larger than that of the $m / z 232.10$ ion peak. Although the $84.15 \mathrm{~m} / z$ ion peak exhibited the greatest area among the peaks of both desmosine and isodesmosine, it was not a favorable ion because peaks with a low $\mathrm{m} / z$ value are often associated with noise resulting from impurities such as peptides or plasticizers. Therefore, the product ions $\mathrm{m} / \mathrm{z} 232.10$ and 397.25 exhibiting high intensity formed from precursor ion $\mathrm{m} / \mathrm{z} 263.65$ were determined to be the best targets for detecting desmosine and isodesmosine. The optimization was also applied to isodesmosine $-{ }^{13} \mathrm{C}_{3},{ }^{15} \mathrm{~N}_{1}$ (Figure S2). Detected ions at $\mathrm{m} / \mathrm{z} 265.65$ and 401.25 corresponded to $\mathrm{m} / \mathrm{z}$ 263.65 and 397.25 peaks of isodesmosine. Optimized MS/MS conditions for desmosine, isodesmosine, and isodesmosine $-{ }^{13} \mathrm{C}_{3},{ }^{15} \mathrm{~N}_{1}$ are summarized in Table $\mathrm{S} 2$.

In order to analyze human plasma samples, calibration curves were drawn for fragment ions $\mathrm{m} / \mathrm{z} 232.10$ and 397.25 with isotopic internal standard isodesmosine $-{ }^{13} \mathrm{C}_{3},{ }^{15} \mathrm{~N}_{1}$. An example calibration sample $(0.005 \mathrm{ppm})$ is shown in Figure S3. As shown in the MS chromatogram, two fragments $(\mathrm{m} / \mathrm{z} 232.10$ and $397.25)$ were clearly observed. In all samples, the retention times of the internal standard and isodesmosine exhibited good reproducibility at approximately $12 \mathrm{~min}$. The peak area ratios between the isotopic standard and isodesmosine were calculated to draw calibration curves (Figures S4 and S5). In 
order to obtain greater accuracy, calibration curves were drawn for each sample group. Satisfactory Rr1 (correlation coefficient value) and Rr2 (coefficient of determination value) indicated that all calibration points were successfully analyzed. Compared with the curve for fragment ion $m / z 232.10$, fragment ion $\mathrm{m} / \mathrm{z} 397.25$ exhibited better accuracy in both stroke and control samples, as the function of fragment ion $m / z 397.20$ was closer to the zero point, and Rr2 was $>0.999$. Therefore, product ion $m / z 397.25$ was selected for the calibration of isodesmosine.

The reproducibility of calibration samples was confirmed using the $m / z 397.25$ ion (Tables 1 and S3). The mean concentration indicates the concentration of a sample as predicated from the calibration curve. Standard deviation, relative standard deviation, and signal-to-noise $(\mathrm{S} / \mathrm{N})$ ratio were also calculated. The limit of quantitation (LOQ) was determined at $\mathrm{S} / \mathrm{N}=10$ unless otherwise stated in Table S3. According to the analytical results, the LOQ of isodesmosine based on the $m / z 397.25$ fragment was $0.005 \mathrm{ppm}$ for stroke samples and $0.01 \mathrm{ppm}$ for healthy control samples. This difference derived from instrument or column conditions, because the two calibration curves were drawn before the respective analyses, which were carried out on different days.

\section{Table 1}

Reproducibility of calibration samples for stroke (fragment: $m / z$ 397.25). 


\begin{tabular}{|c|c|c|c|c|}
\hline Concentration of isodesmosine (ppm) & Area ratio & $\begin{array}{l}\text { Mean concentration } \\
(\mathrm{ppm})\end{array}$ & $\begin{array}{l}\text { Area ratio } \\
\text { SD }\end{array}$ & $\begin{array}{l}\text { Area ratio } \\
\text { RSD }\end{array}$ \\
\hline \multirow[t]{3}{*}{0.005} & 0.0631258 & 0.00917 & \multirow[t]{3}{*}{0.0179} & \multirow[t]{3}{*}{0.4218} \\
\hline & 0.0333533 & 0.00473 & & \\
\hline & 0.030962 & 0.00437 & & \\
\hline \multirow[t]{3}{*}{0.01} & 0.0705102 & 0.01027 & \multirow[t]{3}{*}{0.0074} & \multirow[t]{3}{*}{0.1094} \\
\hline & 0.0589324 & 0.00854 & & \\
\hline & 0.0726177 & 0.01059 & & \\
\hline \multirow[t]{3}{*}{0.02} & 0.139143 & 0.02052 & \multirow[t]{3}{*}{0.0062} & \multirow[t]{3}{*}{0.0456} \\
\hline & 0.128068 & 0.01886 & & \\
\hline & 0.138298 & 0.02039 & & \\
\hline \multirow[t]{3}{*}{0.05} & 0.31623 & 0.04695 & \multirow[t]{3}{*}{0.0094} & \multirow[t]{3}{*}{0.0290} \\
\hline & 0.32687 & 0.04853 & & \\
\hline & 0.335067 & 0.04976 & & \\
\hline \multirow[t]{3}{*}{0.1} & 0.696939 & 0.10377 & \multirow[t]{3}{*}{0.0193} & \multirow[t]{3}{*}{0.0285} \\
\hline & 0.675204 & 0.10052 & & \\
\hline & 0.658539 & 0.09804 & & \\
\hline
\end{tabular}

\section{Measurement of the plasma concentrations of desmosine and isodesmosine in acute stroke patients using isotope- dilution LC-MS/MS}

A total of 17 plasma samples obtained from stroke patients (samples S1-S9) and healthy control subjects (samples C1-C8) were hydrolyzed and purified as previously reported [11-13]. A comparison of the peak area ratios of desmosine and isodesmosine with the internal standard is shown in Table 2. Raw values for fragment ions $m / z 232.10$ and 397.25 are shown in the Supporting Information (Tables S4 and S5). Quantitative analysis of desmosine and isodesmosine was possible for all samples in which the peak area could be determined for both the $\mathrm{m} / \mathrm{z} 232.10$ and 397.25 channels. Therefore, peak areas for desmosine and isodesmosine were obtained for seven samples (S1-S3, S6, S8, S9, and C3). Four samples (S4, S5, S7, and C7) were detected in the $\mathrm{m} / z 397.25$ channel, but they were below the LOQ due to lack of detection of the $\mathrm{m} / z 232.10$ channel. Hence, the peak area ratio of desmosine and isodesmosine in these 
samples (S4, S5, S7, and C7) was above the limit of detection (LOD) but under the LOQ. In summary, the areas of desmosine and isodesmosine in all plasma samples from stroke patients were above the LOD. Quantitative analysis of desmosine and isodesmosine was thus possible for six of nine samples from stroke patients (S1, S2, S3, S6, S8 and S9). Among the eight healthy control group samples, the peak area ratio of desmosine and isodesmosine was above the LOD in two samples (C3 and C7). Quantitative analysis of desmosine and isodesmosine was only possible for one control sample (C3); the other five samples (C1, C2, C4, C5, and C8) were below the LOD.

\section{Table 2}

Peak area ratio of desmosine and isodesmosine compared with the internal standard. 


$\begin{array}{lll}\text { Desmosine Isodesmosine } & \begin{array}{l}\text { Desmosine }+ \\ \text { Isodesmosine }\end{array} \quad \text { Comment }\end{array}$

\begin{tabular}{|c|c|c|c|c|}
\hline S1 & 0.03701812 & 0.031877437 & 0.068895558 & \\
\hline S2 & 0.014672097 & 0.047289901 & 0.061961998 & \\
\hline S3 & 0.024437845 & 0.03809611 & 0.062533955 & \\
\hline S4 & - & - & $<L O Q$ & Fragment 232.15 was ND. \\
\hline S5 & - & - & $<$ LOQ & Fragment 232.15 was ND. \\
\hline S6 & 0.036334632 & 0.017706312 & 0.054040945 & \\
\hline S7 & - & - & $<$ LOQ & Fragment 232.15 was ND. \\
\hline S8 & 0.02580029 & 0.030987129 & 0.056787419 & \\
\hline S9 & 0.02564125 & 0.02081456 & 0.04645581 & \\
\hline C1 & - & - & $<$ LOD & $\begin{array}{l}\text { Fragments } 232.15 / 397.25 \text { were } \\
\text { ND. }\end{array}$ \\
\hline $\mathrm{C} 2$ & - & - & $<L O D$ & $\begin{array}{l}\text { Fragments } 232.15 / 397.25 \text { were } \\
\text { ND. }\end{array}$ \\
\hline C3 & 0.015242721 & 0.031290451 & 0.046533172 & \\
\hline C4 & - & - & $<L O D$ & $\begin{array}{l}\text { Fragments } 232.15 / 397.25 \text { were } \\
\text { ND. }\end{array}$ \\
\hline C5 & - & - & $<$ LOD & $\begin{array}{l}\text { Fragments } 232.15 / 397.25 \text { were } \\
\text { ND. }\end{array}$ \\
\hline C6 & - & - & $<L O D$ & $\begin{array}{l}\text { Fragments } 232.15 / 397.25 \text { were } \\
\text { ND. }\end{array}$ \\
\hline $\mathrm{C7}$ & - & - & $<L O Q$ & Fragment 397.25 was ND. \\
\hline C8 & - & - & $<$ LOD & $\begin{array}{l}\text { Fragments } 232.15 / 397.25 \text { were } \\
\text { ND. }\end{array}$ \\
\hline
\end{tabular}

*All values refer to mean area ratio obtained from calibration curves. S: stroke; C: control; LOQ: limit of quantitation; LOD: limit of detection; ND: not detected.

Based on the peak area ratio, the concentration of desmosine, isodesmosine, and their total amount in plasma were calculated (Figures $2 \& 3$ and Table S6). The relationship between the amount of desmosine and the patient's pathology was also confirmed. Plasma concentrations of desmosine and isodesmosine were elevated in stroke patients compared with healthy volunteers ( $0.05810 \mathrm{vs.} 0.005817$, unpaired $t$-test, $P<0.05$. Figure 3$)$. In contrast, there were no obvious differences in desmosine concentration between ischemic versus hemorrhage stroke patients (representative cases are shown in Figure 4). 
Several issues must be addressed to interpret desmosine and isodesmosine levels in plasma (Figures 1 and 2). First, the number of plasma samples analyzed was small. Second, elevated plasma elastin levels are not solely derived from vascular injuries caused by acute cerebral stroke. A history of COPD and smoking habit can affect elastin dynamics. Plasma concentrations of desmosine and isodesmosine should therefore be carefully interpreted in patients who have a history of COPD or smoking habit. Finally, the optimal time point for measuring plasma elastin levels remains unclear. The progression of a brain injury depends on a variety of factors, such as stroke subtype and location of the stroke. Future studies including larger cohorts should be conducted to validate the findings of the present study.

Based on these results, we conclude that the plasma of stroke patients contains increased levels of desmosine and isodesmosine due to vascular injuries. The present research suggests that desmosine and isodesmosine could be used as novel biomarkers for vascular injuries after acute cerebral stroke. Further studies should be conducted to validate the diagnostic value of desmosine and isodesmosine measurements for evaluating vascular injuries caused by acute cerebral stroke.

\section{Methods}

\section{Study Population and Clinical Data Collection}

Stroke patients were prospectively recruited for this study between December 2018 and February 2019. Patients with a first-ever acute cerebral stroke whose onset was within $24 \mathrm{~h}$ of admission were considered for eligibility in the present study. The diagnosis of stroke was based on brain computed tomography or magnetic resonance imaging. The exclusion criteria were recent history of conditions involving elastic fiber defects such as COPD, asthma, bronchiectasis, interstitial lung disease, cardiovascular diseases, hypertension, diabetes, renal failure, and obesity [37]. Patients with craniotomy, transient ischemic attack, subarachnoid hemorrhage, or functional disability before the index stroke were also excluded.

Nine acute stroke patients were included in the present study. Eight healthy volunteers who had no previous stroke episodes and no remarkable lesions on magnetic resonance imaging were registered. Table 2 shows baseline demographic and other characteristics of acute stroke patients and healthy volunteers. This research was conducted in accordance with the Helsinki Declaration. Research procedures were approved by the Institutional Review Board of Sendai Medical Center (approval number: $29-6$ ). Written informed consent was obtained from each subject's family or relatives before inclusion in the study.

\section{Plasma Isolation}

A total of $5 \mathrm{~mL}$ of peripheral blood was collected into a heparin-containing tube from each participant enrolled in this study. Plasma was isolated by centrifugation of peripheral blood at $1,500 \times g$ at $4^{\circ} \mathrm{C}$ for 10 min. Plasma samples were immediately frozen and preserved in a $-80^{\circ} \mathrm{C}$ freezer until further analysis.

\section{General Experimental Procedure}


All LC-MS/MS analyses were conducted using a Shimadzu LC-20AD, DGU-20A ${ }_{5 R}$, SPD-20A, SIL-20AC, CBM-20A, CTO-20AC, and LCMS-8030 plus system. Nebulizer gas for MS/MS was provided using a $\mathrm{N}_{2}$ Supplier model 24F SLP-07E-S53C (System Instruments, Tokyo, Japan). HPLC for separation was conducted on Shimadzu LC-6AD, DGU-20A 3 , SPD-20A, FCV-12AH, and FRC-10A instruments. The results were collected and analyzed using Shimadzu Labsolution software. Nebulizing gas was set at $2.0 \mathrm{~L} / \mathrm{min}$; $\mathrm{DL}$ temperature was set at $250^{\circ} \mathrm{C}$; heat block temperature was set at $400^{\circ} \mathrm{C}$; drying gas flow was 15 $\mathrm{L} / \mathrm{min}$; CID gas was set at $230 \mathrm{kPa}$; and ion gauge vacuum was stable at $1.72 \times 10^{-3} \mathrm{~Pa}$. Solvents or additives such as MeCN and FA were HPLC grade and purchased from commercial suppliers.

Isodesmosine $-{ }^{13} \mathrm{C}_{3},{ }^{15} \mathrm{~N}_{1}$ was chemically synthesized in our previous research [10]. All columns for HPLC separation were purchased from commercial suppliers, including the Symmetry C18 $(250 \mathrm{~mm} \times 4.6 \mathrm{~mm}$, Waters, MA, USA), YMC-Pack ODS-AM (150 mm×4.6 mm, YMC, Kyoto, Japan), and Supelco Discovery HS F5-3 (3 $\mu \mathrm{m}, 150 \mathrm{~mm} \times 2.1 \mathrm{~mm}$, Sigma Aldrich, St. Louis, MO, USA). A Supelguard guard cartridge ( $3 \mu \mathrm{m}, 2.1$ $\mathrm{mm}$, Sigma Aldrich) was used alongside the Supelco Discovery HS F5-3 column. Terumo syringes ( $1 \mathrm{~mL})$ (Terumo, Tokyo, Japan) and Millex LH filters $(0.45 \mu \mathrm{L})$ (Merck Millipore, MO, USA) were also used.

\section{Hydrolysis of Plasma Samples[11-13]}

Analytical plasma sample (volume of each sample was shown in Table S7) and $1 \mu \mathrm{L}$ of isodesmosine-

${ }^{13} \mathrm{C}_{3},{ }^{15} \mathrm{~N}_{1}(100 \mathrm{ppm})$ were distributed into a small-scale vial with deionized water and an equal amount of $6 \mathrm{~N} \mathrm{HCl}$ aq was added. The vial was tightly capped, then heated to $110^{\circ} \mathrm{C}$ for $24 \mathrm{~h}$ without stirring. The color of plasma samples changed from clear pale yellow to brown with precipitation. These samples were then cooled to $60^{\circ} \mathrm{C}$, and $\mathrm{HCl}$ was completely evaporated by an air pump.

\section{Cellulose Column Preparation [11-13]}

A column to purify hydrolyzed sample was prepared using $130 \mathrm{mg}$ of $5 \%$ CF1 cellulose powder. A $3 \mathrm{~mL}$ mixture of 1-butanol:acetic acid: $\mathrm{H}_{2} \mathrm{O}(4: 1: 1[\mathrm{v} / \mathrm{v} / \mathrm{v}])$ was prepared and then poured into the cellulose. The mixture was stirred for $24 \mathrm{~h}$ at room temperature to obtain a slurry. The slurry was charged into a syringe with a mesh cap and pressure was applied by pushing a pipette rubber to compress.

\section{Purification of an Analytical Plasma Sample [11-13]}

According to previous studies, $1 \mathrm{~mL}$ of premixed solution (1-butanol:acetic acid:deionized water; 4:1:1 $[\mathrm{v} / \mathrm{v} / \mathrm{v}])$ was added in order to dissolve the sample and apply it to the prepared cellulose column. This procedure was repeated twice. After draining, the column was washed three times with $3 \mathrm{~mL}$ of premixture followed by elution of desmosines using deionized water $(3 \mathrm{~mL})$. The water phase was collected and removed using an air pump at $60^{\circ} \mathrm{C}$ for a few hours. Following this procedure, the sample was brought to $201 \mu \mathrm{L}$ in an HPLC vial using deionized water and a Millex filter. Each sample was stored at 
$4^{\circ} \mathrm{C}$, and $20 \mu \mathrm{L}$ of each sample was injected into the LC-MS/MS system using an auto-sampler and analyzed by the optimized method (Tables S1 and S2).

\section{Preparation of Calibration Samples}

Synthetic isodesmosine samples $(0.005,0.01,0.02,0.05$, and $0.1 \mathrm{ppm})$ were prepared in $200 \mu \mathrm{L}$ total volume, and $1 \mu \mathrm{L}$ of isodesmosine $-{ }^{13} \mathrm{C}_{3},{ }^{15} \mathrm{~N}_{1}$ (100 ppm) was added to each sample and mixed. Samples were stored at $4^{\circ} \mathrm{C}$, and $20 \mu \mathrm{L}$ of each sample was injected into the LC-MS/MS system using an autosampler and analyzed by the optimized method summarized in Tables S1 and S2. The calibration curve was drawn twice: before analysis of the stroke samples (samples S1-S9) and before analysis of the control samples (samples C1-C8) in order to reflect instrument conditions.

\section{Optimization of LC-MS/MS conditions}

An LCMS-8030 plus system was used for all LC-MS/MS analyses using a Supelco Discovery HS F5-3 (3 $\mu \mathrm{m}, 150 \mathrm{~mm} \times 2.1 \mathrm{~mm}$ ) column unless otherwise noted. HPLC conditions were set as mobile phase A $(0.1 \% \mathrm{FA}$ in $\mathrm{MeCN})$ and $\mathrm{B}(0.1 \% \mathrm{FA}$ in water) with a programmed linear flow rate to obtain sharp and reproducible peaks (Table $\mathrm{S} 1$ ).

MS/MS conditions were determined by precursor ion search followed by product ion search, which enumerates desirable product ions (Table S2). The precursor ion was set according to the desmosine molecular mass $(m / z=526.3)$. Product ions were searched between $m / z 80$ and $m / z 500$ under conditions in which the minimum intensity was $>1000$ and ion tolerance was 0.5 . After these processes, the precursor ion selected was $m / z 263.65$. For the $m / z 263.65$ ion, some product ions exhibiting high intensity were selected ( $\mathrm{m} / \mathrm{z} 232.10$ and 397.25). As the MS/MS system uses a quadrupole analyzer, the voltages of Q1, Q3, and collision energy were also optimized. Conditions for the analysis of isodesmosine $-{ }^{13} \mathrm{C}_{3},{ }^{15} \mathrm{~N}_{1}(\mathrm{~m} / \mathrm{z}=530.3)$ were also determined as done for desmosine.

Quantitation was performed in MRM mode for desmosines using isodesmosine $-{ }^{13} \mathrm{C}_{3},{ }^{15} \mathrm{~N}_{1}$ as the internal standard. The optimized HPLC and MS/MS conditions were utilized for all analyses. Dwell time (400 msec for internal standard, $50 \mathrm{msec}$ for desmosines) and pause time (1.0 msec) were newly optimized for LC-MS/MS analyses.

\section{Calculations and Data Processing}

In this study, a calculation step was required to determine the concentrations of desmosine and isodesmosine. This calculation would help discriminate inseparable desmosine and isodesmosine and obtain the isodesmosine peak area.

Parameters given by analysis of clinical samples:

$$
\begin{aligned}
& A=[\text { arearatioof } 232.10(\text { inclinicalsample })] \\
& B=[\text { arearatioof397.25(inclinicalsample })]
\end{aligned}
$$


Constants (determined by analysis of a synthetic sample):

$$
\begin{gathered}
C d a=[\text { desmosine'sareaof } 232.10] \\
C d b=[\text { desmosine's sareaof } 397.25] \\
\text { Cia }=[\text { isodesmosine' sareaof } 232.10] \\
C i b=[\text { isodesmosine' sareaof } 397.25]
\end{gathered}
$$

Unknowns:

$$
\begin{gathered}
D a=[\text { arearatioof } 232.10(\text { amountofdesmosineinclinicalsample })] \\
D b=[\text { arearatioof397.25(amountofdesmosineinclinicalsample })] \\
I a=[\text { arearatioof } 232.10(\text { amountofisodesmosineinclinicalsample })] \\
I b=[\text { arearatioof397.25(amountofisodesmosineinclinicalsample })]
\end{gathered}
$$

Equations (1)-(6) below were established for each sample group (stroke or control) and referred the same calibration curves and corresponding instrument conditions. The mixed raw peak contained desmosine and isodesmosine, as indicated in equations (1) and (2).

$$
A=D a+I a \ldots(1), B=D b+I b \ldots(2)
$$

Based on the theory and reproducibility of the LC-MS/MS instrument, for each compound, the area ratio of the product ions should be stable under the same instrument conditions, as shown in equations (3) and (4).

$$
\frac{C d b}{C d a}=\frac{D b}{D a} \ldots(3), \frac{C i b}{C i a}=\frac{I b}{I a} \ldots
$$

When the concentrations of desmosine and isodesmosine are the same, equations (5) and (6) can be obtained.

$$
\frac{C d a}{C i a}=\text { constant } \ldots(5), \frac{C d b}{C i b}=\text { constant } \ldots(6)
$$

The constants ( $C d a, C d b, C i a$, and $C i b$ ) were determined by analysis of synthetic desmosine and isodesmosine (Table S8). Each sample was prepared to $0.01 \mathrm{ppm}$, and the resulting peak area was obtained for the $\mathrm{m} / \mathrm{z} 232.10$ and 397.25 ions. The constants were obtained separately for stroke and control samples, similar to the calibration curves. 
Based on these equations, $I b$ and $D b$ were calculated as shown below. From Eq. (3), Eq. (1) was assigned as follows.

$$
A=\frac{D b \times C d a}{C d b}+I a
$$

Based on (4),

$$
A=\frac{D b \times C d a}{C d b}+\frac{I b \times C i a}{C i b}
$$

and on (2),

$$
A=\frac{(B-I b) \times C d a}{C d b}+\frac{I b \times C i a}{C i b}=I b \times\left(\frac{C i a}{C i b}-\frac{C d a}{C d b}\right)+\frac{B \times C d a}{C d b}
$$

$\therefore$

The area of desmosine should be modified to apply the calibration curve drawn for isodesmosine.

Therefore, the area ratio of desmosine was obtained from $l b$ and converted into an area ratio of the same concentration of isodesmosine using Eq. (6).

$\mathrm{Db}=\backslash$ frac $\{(\mathrm{B}-\mathrm{lb}) \backslash$ times $\mathrm{Cib}\}\{\mathrm{Cdb}\}$

The equations for desmosine and isodesmosine are summarized in equations (7) and (8). The concentrations of isodesmosine $(I b)$, desmosine $(D b)$, and their total concentration $(I b+D b)$ were determined from the calibration curve showing the relationship between isodesmosine's peak area ratio and its concentration compared with the internal standard. The calculated total level, which was determined based on the peak area of the corresponding sample, was above the LOQ for each sample.

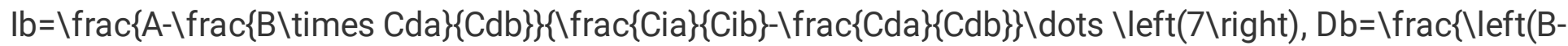

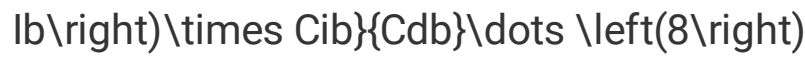

The concentration obtained by the calibration curve was converted into the concentration in plasma. The original volume of plasma samples is noted in Table S7. Because each synthetic isodesmosine sample for calibration $(200 \mu \mathrm{L})$ was diluted to $201 \mu \mathrm{L}$, precise concentration should be considered. Also, all plasma samples were diluted by the addition of internal standard $(1 \mu \mathrm{L})$ and then concentrated to $201 \mu \mathrm{L}$. Therefore, the original concentration in plasma can be described by Eq. (9). It should be noted that according to the isotope-dilution method, the concentration of internal standard was set as the same (1 $\mu \mathrm{L}$ of $100 \mathrm{ppm}$ internal standard was contained in the $201 \mu \mathrm{L}$ sample) between the calibration and plasma samples.

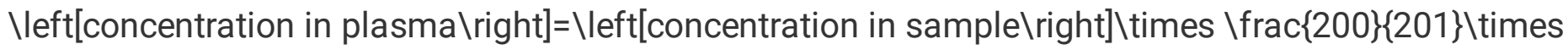

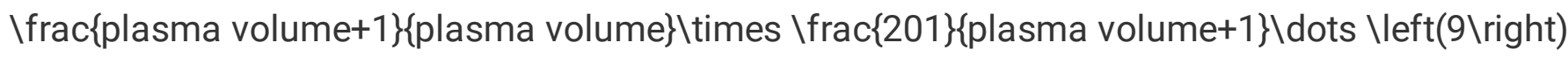
To confirm the discrimination of total concentration, stroke and control sample data were analyzed using an unpaired $t$-test (Fig. 3). Differences were evaluated using a two-sided test with an alpha level of 0.05 . 
Samples S4-S5, S7, C1-C2, and C4-C8, which could not be quantified, were regarded as 0 ppm.

Consequently, as the difference between plasma and healthy samples met the significance level of $5 \%$ ( $P$ $=0.027)$, stroke patients could be discriminated from healthy subjects based on the total concentration of desmosine and isodesmosine.

\section{Statistical Analysis}

Statistical analyses were performed using GraphPad Prism 8.0 (GraphPad Software, Inc., San Diego, CA, USA). A two-sided unpaired $t$-test with an alpha level of 0.05 was conducted to evaluate the difference in plasma desmosine and isodesmosine levels between stroke patients and healthy controls. Samples S4$\mathrm{S} 5, \mathrm{~S} 7, \mathrm{C} 1-\mathrm{C} 2$, and $\mathrm{C} 4-\mathrm{C} 8$, which could not be quantified, were regarded as $0 \mathrm{ppm}$.

\section{Conclusion}

We developed a novel quantitative LC-MS/MS method to analyze desmosine and isodesmosine levels using isotopically labeled internal standard isodesmosine ${ }^{13} \mathrm{C}_{3},{ }^{15} \mathrm{~N}_{1}$. This analytical method provided reliable quantitative measurement of desmosines in human plasma samples with good reproducibility. We measured the concentrations of desmosine and isodesmosine in plasma collected from nine acute cerebral stroke patients and eight healthy controls. The results showed that plasma from acute cerebral stroke patients contains higher desmosine levels than plasma from healthy controls, suggesting the potential value of desmosine and isodesmosine as biomarkers for evaluating the extent of vascular injury after acute cerebral stroke.

\section{Declarations}

\section{Acknowledgements}

This work was supported by the Japan Society for the Promotion of Science (JSPS) through a KAKENHI grant (JP19K09498). Fellowships (to A.M.) from Yoshida Scholarship Foundation and JSPS are also acknowledged. We thank Ms. Manami Kobayashi (Shimadzu Co., Ltd.) for valuable suggestions. Tomo.I. and T.U. thank the Kogyokusha School (Shinagawa, Tokyo, Japan).

\section{Author contributions:}

A.M. established the LC-MS/MS methods and analyzed clinical samples; A.M., R.T., Tomo.I., K.N., and T.U. contributed to manuscript preparation; R.A. analyzed control samples by LC-MS/MS; A.I. conducted preliminary LC-MS/MS experiments; T.Ta. prepared the isotopically labeled compound; Tomo.I., Tomoh.I., Ta.I. collected clinical samples of stroke patients; Tomo.I., K.N., T.To., and T.U. supervised the work; T.U. handled the manuscript. 


\section{Competing Interests Statement}

The authors declare no competing interests.

\section{Additional Information}

Supplementary information accompanies this paper at doi:...

\section{References}

1. Rosenbloom, J., Abrams, W. R. \& Mecham, R. Extracellular matrix 4: The elastic fiber. FASEB J., 7, 1208-1218 (1993).

2. Debelle, L. \& Tamburro, A. M. Elastin: molecular description and function. Int. J. Biochem. Cell. Biol.31, 261-272 (1999).

3. Partridge, S. M., Elsden, D. F. \& Thomas, J. Constitution of the cross-linkages in elastin. Nature, 197, 1297-1298 (1963).

4. Thomas, J., Elsden, D. F. \& Partridge, S. M. Degradation products from elastin: Partial structure of two major degradation products from the cross-linkages in elastin. Nature, 200, 651-652 (1963).

5. Boutin, M., et al. High-sensitivity NanoLC - MS/MS analysis of urinary desmosine and isodesmosine. Anal. Chem., 81, 1881-1887 (2009).

6. Miliotis, T., Lindberg, C., Semb, K. F., van Geest, M. \& Kjellstrom, S. Quantitative high-performance liquid chromatography-tandem mass spectrometry method for the analysis of free desmosines in plasma and urine. J. Chromatogr. A, 1308, 73-78 (2013).

7. O. Albarbarawi, et al. Characterization and validation of an isotope-dilution LC-MS/MS method for quantification of total desmosine and isodesmosine in plasma and serum. Bioanal., 5, 1991-2001 (2013).

8. Lamerz, J., Friedlein, A., Soder, N., Cutler, P. \& Dobeli, H. Determination of free desmosine in human plasma and its application in two experimental medicine studies. Anal. Biochem., 436, 127-136 (2013).

9. Ongay, S. et al. Quantification of free and total desmosine and isodesmosine in human urine by liquid chromatography tandem mass spectrometry: A comparison of the surrogate-analyte and the surrogate-matrix approach for quantitation. J. Chromatogr. A, 1326, 13-19 (2014).

10. Ma, S., Lieberman, S., Turino, G. M. \& Lin, Y. Y. The detection and quantitation of free desmosine and isodesmosine in human urine and their peptide-bound forms in sputum. Proc. Natl. Acad. Sci., U.S.A., 100, 12941-12943 (2003).

11. Ma, S., Lin, Y. Y. \& Turino, G. M. Measurements of desmosine and isodesmosine by mass spectrometry in COPD. Chest, 131, 1363-1371 (2007).

12. Ma, S., Turino, G. M. \& Lin, Y. Y. Quantitation of desmosine and isodesmosine in urine, plasma, and sputum by LC-MS/MS as biomarkers for elastin degradation. J. Chromatogr. B, 879, 1893-1898 (2011). 
13. Ma, S., Turino, G. M., Hayashi, T., Yanuma, H., Usuki, T. \& Lin, Y. Y. Stable Deuterium Internal Standard for the Isotope-Dilution LC-MS/MS Analysis of Elastin Degradation. Anal. Biochem., 440, 158-165 (2013).

14. Huang, J. T.-J. et al., Clinical validity of plasma and urinary desmosine as biomarkers for chronic obstructive pulmonary disease. Thorax, 67, 502-508 (2012).

15. Wieling, J. LC-MS-MS experiences with internal standards. Chromatographia, 55, S107-S113 (2002).

16. Suzuki, R., Yanuma, H., Hayashi, T., Yamada, H. \& Usuki, T. Syntheses of natural and deuterated desmosines via palladium-catalyzed cross-coupling reactions. Tetrahedron, 71, 1851-1862 (2015).

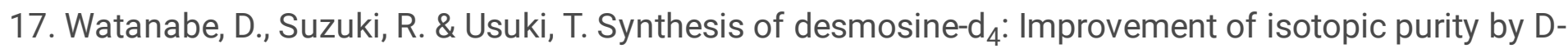
H exchange of amino groups. Tetrahedron Lett., 58, 1194-1197 (2017).

18. Gosetti, F., Mazzucco, E., Zampieri, D. \& Gennaro, M. C. Signal suppression/enhancement in highperformance liquid chromatography tandem mass spectrometry. J. Chromatogr. A, 1217, 3929-3937 (2010).

19. Tanigawa, T., Komatsu, A. \& Usuki, T. $\left[{ }^{13} \mathrm{C}_{3},{ }^{15} \mathrm{~N}_{1}\right]$-Labeled isodesmosine: a potential internal standard for LC-MS/MS analysis ofdemosines in elastin degradation. Bioorg. Med. Chem. Lett., 25, 20462049 (2015).

20. Usuki, T., Sugimura, T., Komatsu, A. \& Koseki, Y. Biomimetic Chichibabin pyridine synthesis of the COPD biomarkers and elastin cross-linkersisodesmosine and desmosine. Org. Lett., 16, 1672-1675 (2014).

21. Tanaka, N., Kurita, M. Murakami, Y. \& Usuki, T. Chichibabin and IsoChichibabin pyridinium syntheses of isodesmosine, desmosine, and their derivatives. Eur. J. Org. Chem., 21, 6002-6009 (2018).

22. Campbell, B. C. V. et al. Ischaemic stroke. Nat Rev Dis Primers, 5, 70 (2019).

23. Macdonald, R. L. \& Schweizer, T. A. Spontaneous subarachnoid haemorrhage. Lancet, 389, 655-666 (2017).

24. Cordonnier, C., Demchuk, A., Ziai, W. \& Anderson, C. S. Intracerebral haemorrhage: current approaches to acute management. Lancet, 392, 1257-1268 (2018).

25. Hankey, G. J. Stroke. Lancet, 389, 641-654 (2017).

26. Powers W. H. et al. Guidelines for the early management of patients with acute ischemic stroke: 2019 Update to the 2018 guidelines for the early management of acute ischemic stroke: A guideline for healthcare professionals from the American Heart Association/Ameican Stroke Association. Stroke50, e344-e418 (2019).

27. Macdonald R.L. \& Schweizer T. A. Spontaneous subarachnoid haemorrhage. Lancet389, 655-666 (2017).

28. Cordonnier C., Demchuk A., Zhai W. \& Anderson C. S. Intracerebral haemorrhage: current approaches to acute management. Lancet392, 1257-1268 (2018).

29. Keep, R. F., Hua, Y. \& Xi, G. Intracerebral haemorrhage: mechanisms of injury and therapeutic targets. Lancet Neuro/11, 720-731 (2012).

30. Rabinstein A. A. Secondary brain injury after aneurysmal subarachnoid haemrrhgae: more than vasospasm. Lancet Neuro/10, 593-595 (2011). 
31. Ng G. J. L. et al. Stroke biomarkers in clinical practice: a critical appraisal. Neurochem. Int.107, 1122, (2017).

32. Simpkins A, et al. Biomarker application for precision medicine in stroke. Transl. Stroke Res.11, 615627, (2020).

33. Sigma Aldrich (Millipore Sigma) HPLC Discovery HS F5 (Jul/27/2021):

https://www.sigmaaldrich.com/analytical-chromatography/hplc/columns/discovery-hplc/hs-f5.html

34. Gross. H. J. Mass Spectrometry: A Textbook, Springer2017.

35. Shimadzu Application Data Sheet No.98 Automatic Optimization of Transitions and Collision Energies (Jul/27/2021): https://solutions.shimadzu.co.jp/an/n/en/gcms/jpo214019.pdf

36. Schräder, C. U., Heinz, A., Majovsky, P. \& Schmelzer, C. E. H. Fingerprinting desmosine-containing elastin peptides, J. Am. Soc. Mass Spectrom.26, 762-773 (2015).

37. Cocciolone, A. J., Hawes, J. Z., Staiculescu, M. C., Johnson, E. O., Murshed, M. \& Wagenseil, J. E. Elastin, arterial mechanics, and cardiovascular disease. Am J Physiol Heart Circ Physiol, 315, H189H205 (2018).

\section{Figures}

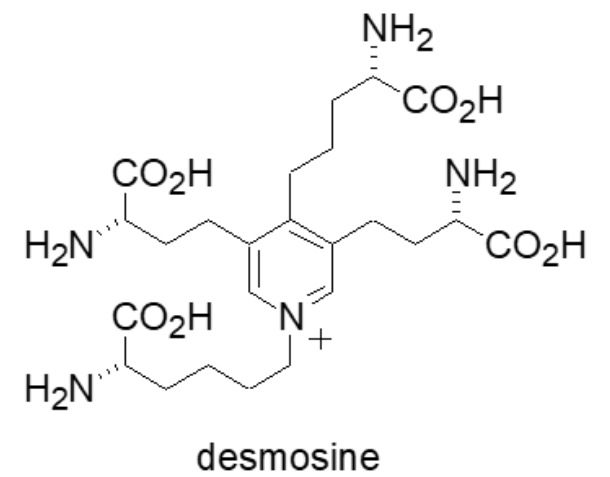

desmosine<smiles>N[C@@H](CCCC[n+]1cc(CC[C@H](N)C(=O)O)cc(CC[C@H](N)C(=O)O)c1CCC[C@H](N)C(=O)O)C(=O)O</smiles>

isodesmosine

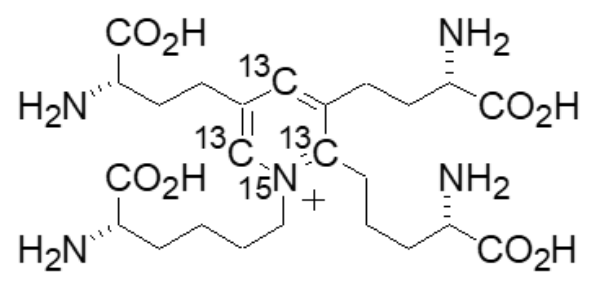

isodesmosine $-{ }^{13} \mathrm{C}_{3},{ }^{15} \mathrm{~N}_{1}$

\section{Figure 1}

Structures of desmosine, isodesmosine, and isodesmosine-13C3, $15 \mathrm{~N} 1$. 


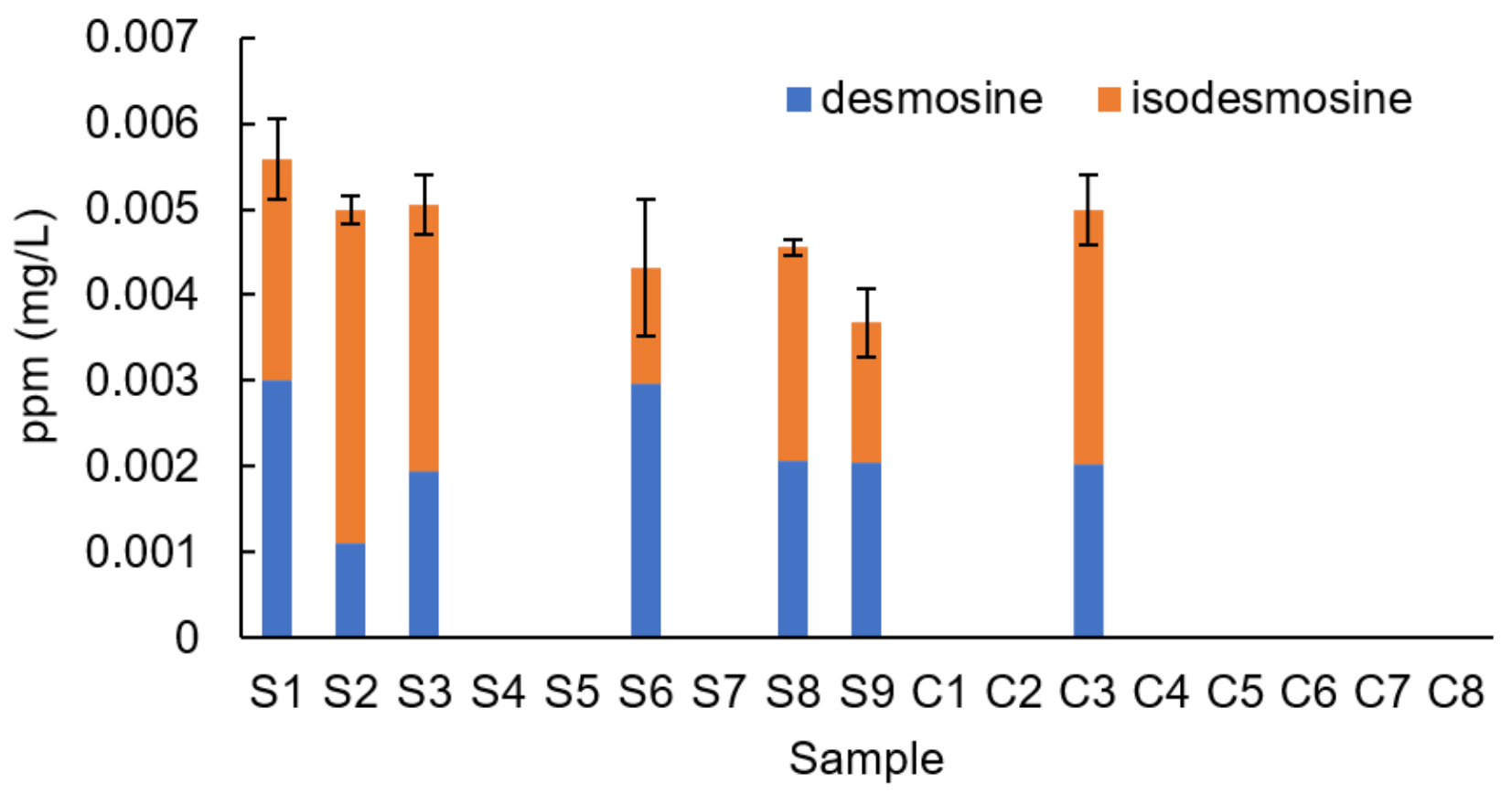

Figure 2

Concentrations of desmosine and isodesmosine in plasma ( $n=3)$. S: stroke; C: control. 


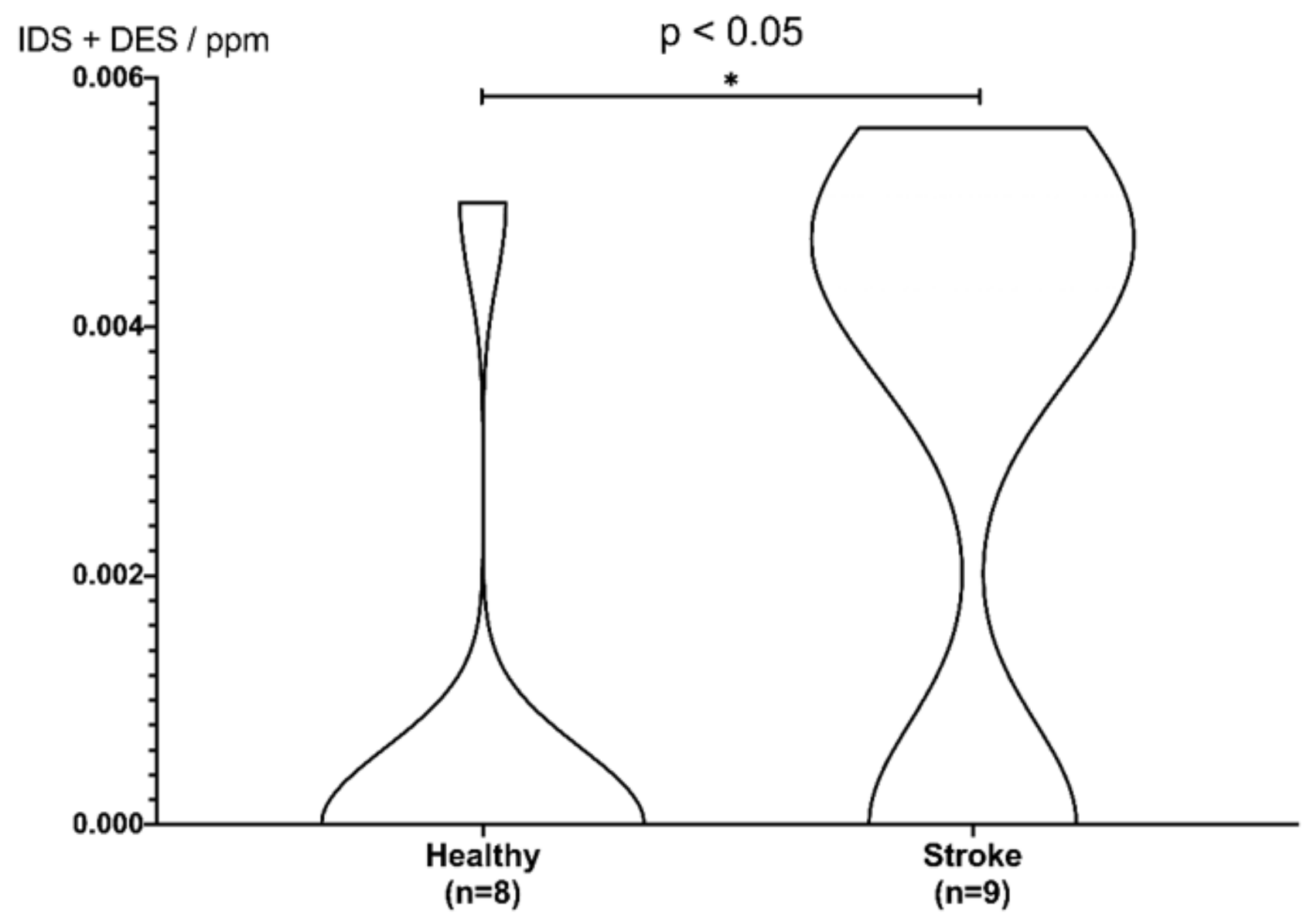

Figure 3

Violin plot for discrimination of stroke patients. Differences in concentrations of desmosine and isodesmosine were analyzed using a two-sided unpaired t-test. 

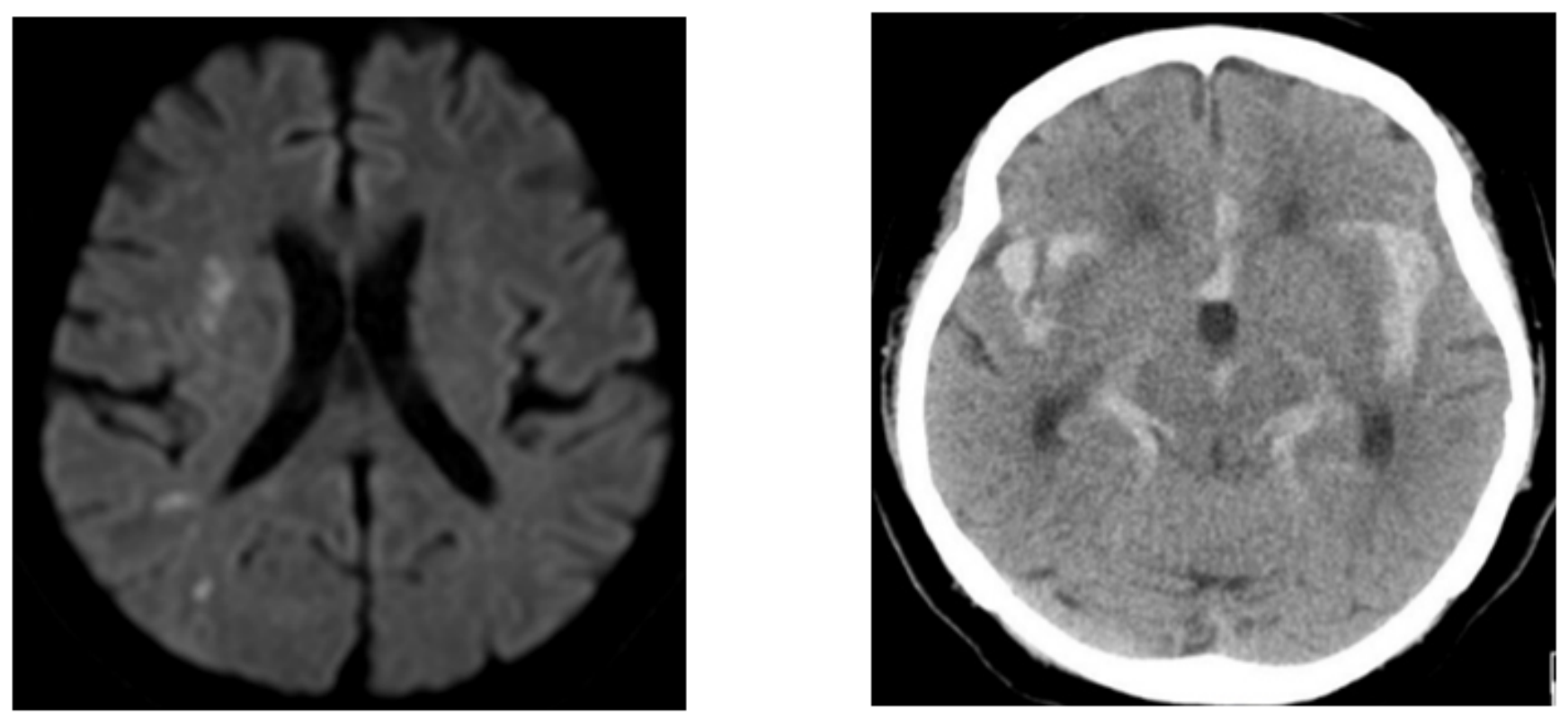

\section{Figure 4}

Representative cases of stroke. Left: A 55-year-old man (S6) with cerebral infarction containing 0.00432 ppm of desmosine. Diffusion-weighted magnetic resonance imaging demonstrated multiple highintensity spots indicating small infarctions. Right: A 77-year-old woman (S9) with subarachnoid hemorrhage containing $0.00367 \mathrm{ppm}$ of desmosine. Computed tomography revealed diffuse hemorrhaging in the basal cisterns.

\section{Supplementary Files}

This is a list of supplementary files associated with this preprint. Click to download.

- SIStrokeDES.pdf 